\title{
Optimal stopping investment in a logarithmic utility-based portfolio selection problem
}

\author{
Xun $\mathrm{Li}^{1 *} \mathbb{D}$, Xianping $\mathrm{Wu}^{2}$ and Wenxin Zhou ${ }^{1}$
}

\section{${ }^{*}$ Correspondence:}

malixun@polyu.edu.hk

'Department of Applied

Mathematics, The Hong Kong

Polytechnic University, Hong Kong,

China

Full list of author information is

available at the end of the article

\begin{abstract}
Background: In this paper, we study the right time for an investor to stop the investment over a given investment horizon so as to obtain as close to the highest possible wealth as possible, according to a Logarithmic utility-maximization objective involving the portfolio in the drift and volatility terms. The problem is formulated as an optimal stopping problem, although it is non-standard in the sense that the maximum wealth involved is not adapted to the information generated over time.

Methods: By delicate stochastic analysis, the problem is converted to a standard optimal stopping one involving adapted processes.

Results: Numerical examples shed light on the efficiency of the theoretical results.

Conclusion: Our investment problem, which includes the portfolio in the drift and volatility terms of the dynamic systems, makes the problem including multi-dimensional financial assets more realistic and meaningful.
\end{abstract}

Keywords: Optimal stopping, Path-dependent, Stochastic differential equation (SDE), Time-change, Portfolio selection

\section{Background}

Optimal stopping problems, a kind of dynamic optimization problems allowing investors to stop investment any time before the maturity in order to maximize their profits or minimize their costs, are of great interest and of importance in various fields such as science, engineering, economics, management, and particularly in financial investment. In reality, choosing a proper time point to stop investment is of importance to hedge risk and to realize maximum return for investors. In practice, it is extremely hard to find the point at which the realized return is maximized, and therefore the investor tries to sell at a price which is as close to the maximum as possible. To help determine this time point, researchers have made significant effort toward the theory of optimal stopping, and Shiryaev et al. (2008) is one of the typical representatives along this line of research. In the field of mathematical finance, furthermore, optimal stopping has been extensively studied for pricing American-style options, which allow option holders to exercise the options before or at the maturity.

The theory of optimal stopping developed in pricing American options can be further applied to determine an optimal stopping point so as to maximize return from financial

(c) The Author(s). 2017 Open Access This article is distributed under the terms of the Creative Commons Attribution 4.0 International License (http://creativecommons.org/licenses/by/4.0/), which permits unrestricted use, distribution, and reproduction in any medium, provided you give appropriate credit to the original author(s) and the source, provide a link to the Creative Commons license, and indicate if changes were made. 
investment for economic agents. Nevertheless, it is extremely hard to let investors realize the highest return, and therefore, the objective is to minimize the distance between the time point at which the investment is stopped and that at which the maximum return can be realized. For example, Ceci and Bassan (2004) study the mixed optimal stopping and stochastic control problems with semicontinuous final reward for diffusion processes and give some properties of the value function. Dayanik and Karatzas (2003) investigate the optimal stopping problems for one dimensional diffusions and showed how to reduce the discounted optimal stopping problem for an arbitrary diffusion process to an undiscounted one for standard Brownian motion. Choi et al. (2004) study an investor's decision to switch from active portfolio management to passive management and modelled it to a mixture of a consumption-portfolio selection problem and an optimal stopping problem. Chang et al. (2009) consider the optimal stopping problem for stochastic differential equations with random coefficients. Shiryaev et al. (2008) address the optimal stopping issue in an equity market by considering a log-normal price process.

The mean-variance approach originated by Markowitz $(1952,1959)$ has been a cornerstone of asset allocation, investment analysis and risk management. In this literature, Merton's $(1969,1971,1973)$ seminal work is considered a benchmark on continuoustime portfolio selection. The single period model is extended by $\mathrm{Li}$ and $\mathrm{Ng}$ (2000) for multi-period case and developed by Zhou and $\mathrm{Li}$ (2000) for continuous-time one, respectively. The work of Li and Zhou (2006) reveals the high opportunity of a Markowitz mean-variance strategy hitting the expected return target before the maturity date. Naturally, investors also hope to decide when to stop the investment over a given investment horizon so as to maximize their profits. This idea has been further developed to determine the optimal selling time for one stock by Shiryaev et al. (2008), who determined a time point at which investors can sell risky assets as close to the maximum return as possible. This again highlights the efficiency of the mean-variance analysis in the field of investment and portfolio selection. Naturally, an investor also hopes to know the time point to stop the investment over a given investment horizon so as to maximize the profit.

In this paper, we devote to choosing an optimal point at which an investor stops the investment among multi assets for gaining maximum benefit. The investor is expected to maximize her personal utilities and to minimize the difference between the realized return at the stopping point and her potentially maximum return. Compared with the work of Shiryaev et al. (2008), we consider the utility function of a quadratic form instead of a relative error criterion. And since multi financial assets are considered, the drift and volatility terms involve the portfolio. These make our analysis more realistic and meaningful.

The rest of the paper is organized as follows. In "Formulation" section, we formulate the problem to a two-stage problem in which the investor is expected to maximize her personal utilities and to minimize the difference between the realized return/wealth at the stopping point and her potentially maximum return/wealth. We first derive the optimal portfolio of the sub-problem by stochastic controls methodology. Then substituting the optimal portfolio into the dynamic systems, we have the optimal stopping time problem with the wealth process without the control variable in the drift and volatility terms. Using time-change technique, the nonadapted problem is transformed into a standard optimal stopping problem. Numerical examples are presented in "Methods" section 
to demonstrate the theoretical results. "Results and discussion" section 4 concludes the work. Some technique details are relegated to an Appendix.

\section{Formulation}

Throughout this paper $\left(\Omega, \mathcal{F}, P,\left\{\mathcal{F}_{s}\right\}_{s \geq 0}\right)$ is a fixed filtered complete probability space on which defined a standard $\mathcal{F}_{s}$-adapted $m$-dimensional Brownian motion $\{W(s), s \geq 0\}$ with $W(0)=0$, and $\widehat{T}>0$ is given and fixed, representing the terminal time of an investment horizon.

There is a financial market in which $m+1$ securities (or assets) are traded continuously. One of the securities is a risk-free asset, whose price follows

$$
\left\{\begin{aligned}
d S_{0}(t) & =r S_{0}(t) d t, \quad t \geq 0 \\
S_{0}(0) & =s_{0}>0
\end{aligned}\right.
$$

where $r>0$ is the interest rate. The other $m$ securities are risky assets, whose prices follows

$$
\left\{\begin{aligned}
d S_{i}(t) & =S_{i}(t)\left\{b_{i} d t+\sum_{j=1}^{m} \sigma_{i j} d W^{j}(t)\right\}, t \geq 0, \\
S_{i}(0) & =s_{i}>0, \quad i=1,2, \cdots, m,
\end{aligned}\right.
$$

where $b:=\left(b_{1}, b_{2}, \cdots, b_{m}\right)^{\prime}$ is the appreciation rate, $\sigma:=\left(\sigma_{i j}\right)_{m \times m}$ is the volatility, and $\sigma^{\prime} \sigma$ is positive definite.

Consider an agent, with an initial endowment $x_{0}>0$ and whose total wealth at time $s \in[0, \hat{T}]$ is denoted by $x(s)$. Assume that the trading of shares is self-financed and takes place continuously, and that transaction cost and consumptions are ignored. Then $x(\cdot)$ satisfies

$$
\left\{\begin{aligned}
d x(s) & =\left\{r x(s)+\sum_{i=1}^{m}\left(b_{i}-r\right) \pi_{i}(s)\right\} d s+\sum_{j=1}^{m} \sum_{i=1}^{m} \sigma_{i j} \pi_{i}(s) d W^{j}(s), \quad 0 \leq s \leq \hat{T}, \\
x(0) & =x_{0},
\end{aligned}\right.
$$

where $\pi_{i}(s), \quad i=1,2 \cdots, m$, denotes the total market value of the agent's wealth in the $i$-th stock. We call the process $\pi(s):=\left(\pi_{1}(s), \pi_{2}(s), \cdots, \pi_{m}(s)\right)^{\prime}$ a portfolio of the agent.

Define the running maximum wealth process

$$
M(s)=\max _{0 \leq u \leq s} x(u), s \geq 0 .
$$

Assume that an investor can stop investment at any point before a pre-specified date $\widehat{T}>0$. The question is to choose an optimal portfolio and to determine the right time to stop investment. The main objective of this study is to determine conditions for which the investor should sell her shares. Ideally, the investor would like to exit when the value is highest, which is at time $s$, such that $x(s)=\alpha M(\widehat{T})$. More generally, the investor may have an investment target that is a fraction of (or possibly equal to) the maximum value, $\alpha M(\widehat{T})$, where $0<\alpha \leq 1$. With this objective, we assume that the investor chooses an exit time to minimize the mean squared difference between exit value and investment target value. We formulate it to the following optimal stopping problem:

$$
\begin{aligned}
\min _{0 \leq \hat{\tau} \leq \widehat{T}} & \mathbb{E}[x(\hat{\tau})-\alpha M(\widehat{T})]^{2}, \\
\text { subject to } & \left\{\begin{array}{cl}
\max _{\pi(\cdot)} & \mathbb{E}[\ln (x(\widehat{T}))], \\
\text { subject to }(x(\cdot), \pi(\cdot)) \text { satisfy (3). }
\end{array}\right.
\end{aligned}
$$


Note that the above two-stage problem setting is very insightful. It is more realistic than those addressed in Shiryaev et al. (2008) since $m$-dimensional financial assets are considered and the drift and volatility terms involving the portfolio.

\section{Methods}

Before further developing techniques derived in Shiryaev et al. (2008), we know the optimal portfolio of sub-problem (5) via stochastic control method

$$
\hat{\pi}(s) \equiv\left(\hat{\pi}_{1}(s), \hat{\pi}_{2}(s), \cdots, \hat{\pi}_{m}(s)\right)^{\prime}=\left(\sigma \sigma^{\prime}\right)^{-1}(b-r \mathbf{1}) x(s),
$$

where $\mathbf{1}=(1,1, \cdots, 1)^{\prime}$ is an $m$-dimensional column vector.

Substituting (6) into (5) yields the wealth process $x(\cdot)$ without the control variable in the drift and volatility terms

$$
\left\{\begin{array}{c}
d x(s)=x(s)\left\{\left(r+|\theta|^{2}\right) d s+\theta^{\prime} d W(s)\right\}, \\
x(0)=x_{0}
\end{array}\right.
$$

where $\theta=\sigma^{-1}(b-r \mathbf{1})$.

This is similar to the case in Shiryaev et al. (2008), but it is more mathematically complex. By virtue of a time-change technique, there exists a one-dimensional standard Brownian motion $B(s), s \geq 0$, on $(\Omega, \mathcal{F}, P)$ such that

$$
\theta^{\prime} W(s)=B(\beta(s)), \quad 0 \leq s \leq \hat{T},
$$

where $\beta(s):=|\theta|^{2} s$.

$$
\text { Set } t:=|\theta|^{2} s \text {, Eq. (7) is equivalent to }
$$

$$
\left\{\begin{aligned}
d x(t) & =x(t)\{\mu d t+d B(t)\} \\
x(0) & =x_{0}
\end{aligned}\right.
$$

where $\mu=\frac{r}{|\theta|^{2}}+1$. Thus, the problem (4) is equivalent to

$$
\min _{0 \leq \tau \leq T} \mathbb{E}[x(\tau)-\alpha M(T)]^{2}
$$

over $\tau \in \mathcal{T}$, the set of all $\mathcal{F}_{t}$-stopping time $\tau \in[0, T]$, where $T=|\theta|^{2} \widehat{T}$. Consequently, the value function associated with problem (9) is

$$
\begin{aligned}
V(t, x, M) & =\min _{t \leq \tau \leq T} \mathbb{E}\left[(x(\tau)-\alpha M(T))^{2} \mid \mathcal{F}_{t}\right] \\
& =\min _{t \leq \tau \leq T} \mathbb{E}\left[x(\tau)^{2}-2 \alpha x(\tau) M(T)+\alpha^{2} M(T)^{2} \mid \mathcal{F}_{t}\right] \\
& =\min _{t \leq \tau \leq T} \mathbb{E}\left[x(\tau)^{2}-2 \alpha x(\tau) \mathbb{E}\left[M(T) \mid \mathcal{F}_{\tau}\right]+\alpha^{2} \mathbb{E}\left[M(T)^{2} \mid \mathcal{F}_{\tau}\right] \mid \mathcal{F}_{t}\right]
\end{aligned}
$$

Defining $v:=\mu-\frac{1}{2}$, we rewrite

$$
x(t):=x(0) \exp (v t+B(t)), \quad M(t):=x(0) \exp \left(\max _{0 \leq u \leq t}(v u+B(u))\right) .
$$


Denote $\psi(t, x(t), M(t))=\mathbb{E}\left[M(T) \mid \mathcal{F}_{t}\right]$ and $\phi(t, x(t), M(t))=\mathbb{E}\left[M(T)^{2} \mid \mathcal{F}_{t}\right]$. Then

$$
\begin{aligned}
\psi & (t, x(t), M(t))=\mathbb{E}\left[M(T) \mid \mathcal{F}_{t}\right] \\
= & \mathbb{E}\left[x(0) \exp \left(\max _{0 \leq u \leq T}(v u+B(u))\right) \mid \mathcal{F}_{t}\right] \\
= & \mathbb{E}\left[x(0) \exp \left(\max \left\{\max _{0 \leq u \leq t}(v u+B(u)), \max _{t \leq u \leq T}(v u+B(u))\right\}\right) \mid \mathcal{F}_{t}\right] \\
= & \mathbb{E}\left[x(0) \exp \left(\max \left\{\max _{0 \leq u \leq t}(v u+B(u)),(v t+B(t))+\max _{0 \leq u \leq T-t}(v u+B(u))\right\}\right) \mid \mathcal{F}_{t}\right] \\
= & \mathbb{E}\left[x(t) \exp \left(\max \left\{\max _{0 \leq u \leq t}(v u+B(u))-(v t+B(t)), \max _{0 \leq u \leq T-t}(v u+B(u))\right\}\right) \mid \mathcal{F}_{t}\right] \\
= & \mathbb{E}\left[x(t) \exp \left(\max \left\{y, \max _{0 \leq u \leq T-t}(v u+B(u))\right\}\right) \mid y=\max _{0 \leq u \leq t}(v u+B(u))-(v t+B(t))\right] \\
= & x(t) G_{1}\left(t, \ln \left(\frac{M(t)}{x(t)}\right)\right),
\end{aligned}
$$

where

$$
G_{1}(t, y)=\mathbb{E}\left[\exp \left(\max \left\{y, \max _{0 \leq u \leq T-t}(v u+B(u))\right\}\right)\right], \quad(t, y) \in[0, T] \times[0, \infty)
$$

and

$$
\begin{aligned}
\phi( & t, x(t), M(t))=\mathbb{E}\left[M(T)^{2} \mid \mathcal{F}_{t}\right] \\
& =\mathbb{E}\left[x(0)^{2} \exp \left(\left(\max _{0 \leq u \leq T}(v u+B(u))\right)^{2}\right) \mid \mathcal{F}_{t}\right] \\
& =\mathbb{E}\left[x(0)^{2} \exp \left(\left(\max \left\{\max _{0 \leq u \leq t}(v u+B(u)), \max _{t \leq u \leq T}(v u+B(u))\right\}\right)^{2}\right) \mid \mathcal{F}_{t}\right] \\
& =\mathbb{E}\left[x(0)^{2} \exp \left(\left(\max \left\{\max _{0 \leq u \leq t}(v u+B(u)),(v t+B(t))+\max _{0 \leq u \leq T-t}(v u+B(u))\right\}\right)^{2}\right) \mid \mathcal{F}_{t}\right] \\
& =\mathbb{E}\left[x(t)^{2} \exp \left(\left(\max \left\{\max _{0 \leq u \leq t}(v u+B(u))-(v t+B(t)), \max _{0 \leq u \leq T-t}(v u+B(u))\right\}\right)^{2}\right) \mid \mathcal{F}_{t}\right] \\
& =\mathbb{E}\left[x(t)^{2} \exp \left(\left(\max \left\{y, \max _{0 \leq u \leq T-t}(v u+B(u))\right\}\right)^{2}\right) \mid y=\max _{0 \leq u \leq t}(v u+B(u))-(v t+B(t))\right] \\
& =x(t)^{2} G_{2}\left(t, \ln \left(\frac{M(t)}{x(t)}\right)\right),
\end{aligned}
$$

where

$$
G_{2}(t, y)=\mathbb{E}\left[\exp \left(\left(\max \left\{y, \max _{0 \leq u \leq T-t}(v u+B(u))\right\}\right)^{2}\right)\right], \quad(t, y) \in[0, T] \times[0, \infty) .
$$

It follows (10) that

$$
V(t, x, M)=\min _{t \leq \tau \leq T} \mathbb{E}\left[x(\tau)^{2}-2 \alpha x(\tau) \psi(\tau, x(\tau), M(\tau))+\alpha^{2} \phi(\tau, x(\tau), M(\tau)) \mid \mathcal{F}_{t}\right],
$$

which is governed by

$$
\left\{\begin{array}{l}
\max \left\{\mathscr{L} V, V-x^{2}+2 \alpha x \psi-\alpha^{2} \phi\right\}=0, \\
V_{M}(t, M, M)=0, \\
V(T, x, M)=(x-\alpha M)^{2},
\end{array}\right.
$$

where the operator $\mathscr{L}$ is defined by

$$
\mathscr{L} f(t, x, M)=f_{t}(t, x, M)+\mu x f_{x}(t, x, M)+\frac{1}{2} x^{2} f_{x x}(t, x, M) .
$$

The value function $V(t, x, M)$ satisfies

$$
V(t, \lambda x, \lambda M)=\lambda^{2} V(t, x, M)
$$


because scaling both $x(t)$ and $M(t)$ by the same positive constant at a time $t$ prior to the terminal time $T$ results in the payoff $(x(T)-\alpha M(T))^{2}$ being scaled by the same constant. In particular, if

$$
U(t, \ln z)=V(t, 1, z), \quad 0 \leq t \leq T, \quad z \geq 1,
$$

then we may determine $V(t, x, M)$ as

$$
V(t, x, M)=x^{2} V\left(t, 1, \frac{M}{x}\right)=x^{2} U\left(t, \ln \left(\frac{M}{x}\right)\right), \quad 0 \leq t \leq T, \quad 0<x \leq M .
$$

According to Eq. (13) and expressions of $G_{1}$ and $G_{2}$, we have

$$
\begin{aligned}
V(t, x, M) & =\min _{t \leq \tau \leq T} \mathbb{E}\left[x(\tau)^{2}-2 \alpha x(\tau) \psi(\tau, x(\tau), M(\tau))+\alpha^{2} \phi(\tau, x(\tau), M(\tau)) \mid \mathcal{F}_{t}\right] \\
& =\min _{t \leq \tau \leq T} \mathbb{E}\left[x(\tau)^{2}-2 \alpha x(\tau)^{2} G_{1}\left(\tau, \ln \left(\frac{M(\tau)}{x(\tau)}\right)\right)+\alpha^{2} x(\tau)^{2} G_{2}\left(\tau, \ln \left(\frac{M(\tau)}{x(\tau)}\right)\right) \mid \mathcal{F}_{t}\right] \\
& =\min _{t \leq \tau \leq T} \mathbb{E}\left[x(\tau)^{2}\left(1-2 \alpha G_{1}\left(\tau, \ln \left(\frac{M(\tau)}{x(\tau)}\right)\right)+\alpha^{2} G_{2}\left(\tau, \ln \left(\frac{M(\tau)}{x(\tau)}\right)\right)\right) \mid \mathcal{F}_{t}\right] \\
& =\min _{t \leq \tau \leq T} \mathbb{E}\left[x(\tau)^{2} G\left(\tau, \ln \left(\frac{M(\tau)}{x(\tau)}\right)\right) \mid \mathcal{F}_{t}\right],
\end{aligned}
$$

where $G(t, y)=1-2 \alpha G_{1}(t, y)+\alpha^{2} G_{2}(t, y)$.

Equation (15) implies that Eq. (9) is equivalent to a standard optimal stopping problem with a terminal payoff $G$ and an underlying (adapted) state process

$$
Y(t)=\ln \left(\frac{M(t)}{x(t)}\right), \quad Y(0)=0 .
$$

Following the dynamic programming approach we consider the problem below

$$
U(t, y)=\inf _{\tau \in \mathcal{T}_{T-t}} \mathbb{E}_{t, y}[G(t+\tau, Y(t+\tau))],
$$

where $Y(t)=y$ under the probability $\mathbb{P}_{t, x}$ with $(t, y) \in[0, T] \times[0, \infty)$ given and fixed, and $\mathcal{T}_{s}$ in general denotes the set of all $\mathcal{F}$-stopping times $\tau \in[0, s]$ for $s>0$.

In fact, $U$ satisfies the following dynamic programming equation (or variational inequalities)

$$
\left\{\begin{array}{cl}
\max \quad & \{\widehat{\mathscr{L}} U, U-G\}=0,(t, y) \in[0, T] \times[0, \infty), \\
\text { subject to } & U_{y}(t, 0+)=0, \quad t \in[0, T), \\
& U(T, y)=G(T, y), y \in(0, \infty),
\end{array}\right.
$$

where the operator $\widehat{\mathscr{L}}$ is defined by

$$
\widehat{\mathscr{L} f}(t, y)=f_{t}(t, y)-(v+2) f_{y}(t, y)+\frac{1}{2} f_{y y}(t, y)+2(v+1) f(t, y) .
$$

Hence, the original problem is transferred into finding $U$. Since $x(\cdot)$ has stationary independent increments and $Y(\cdot)$ is a Markovian process, we rewrite

$$
U(t, y)=\inf _{0 \leq \tau \leq T-t} \mathbb{E}\left[G\left(t+\tau, Y^{y}(\tau)\right)\right],
$$

where $Y(\cdot)$ under $\mathbb{P}$ is explicitly given as

$$
Y^{y}(t)=y \vee \ln \left(\frac{M(t)}{x(t)}\right), \quad t \geq 0 .
$$

Theoretically, we have derived a region in which the venture capitalist may sell the shares they hold, given the pre-determined relationship between her target return and the expected maximum return. 
Theorem 1 The holding region is

$$
C=\{(t, y) \in[0, T] \times[0, \infty): U(t, y)<G(t, y)\},
$$

while the exit region is

$$
D=\{(t, y) \in[0, T] \times[0, \infty): U(t, y)=G(t, y)\} .
$$

Also, an optimal exit time is

$$
\tau^{*}=\inf \left\{t \in[0, T]:\left(t, \ln \left(\frac{M(t)}{x(t)}\right)\right) \in D\right\} .
$$

\section{Results and discussion}

To investigate comparative statics, we present one numerical example in which we change the value of the parameter $\alpha$. Following the standard approach for estimating the above problem via the finite difference approach, we solve the mathematical formulation given in Eq. (16) by imposing a uniform grid on the $(t, y)$ domain. A Crank-Nicolson scheme is adopted for the discretization of the partial differential equation and the semi-infinite interval for $y$ is truncated at a sufficiently large value of $y$. The derivative boundary condition is discretized using a forward difference approximation. For the results shown below, we take the grid spacing to be 0.005 for $y$ and 0.001 for $t$ dimensions.

Let $m=3$. The interest rate of the bond and the appreciation rate of the $m$ stocks are $r=0.05$ and $\left(b_{1}, b_{2}, b_{3}\right)^{\prime}=(0.1,0.12,0.15)^{\prime}$, respectively, and the volatility matrix is

$$
\sigma=\left[\begin{array}{ccc}
0.3000 & 0 & 0 \\
0.2000 & 0.3464 & 0 \\
0.2500 & 0.1443 & 0.4082
\end{array}\right] \text {. }
$$

Then

$$
\theta:=\sigma^{-1}\left(b_{1}-r, b_{2}-r, b_{3}-r\right)^{\prime}=(0.1667,0.1058,0.1055)^{\prime} .
$$

Using Theorem 1 and the parameter value of $\alpha$ ranging between 0.8 and 1 , we observe that the exit region decreases as the value of $\alpha$ increases, as shown by the combined picture at the right-bottom corner of Fig. 1.

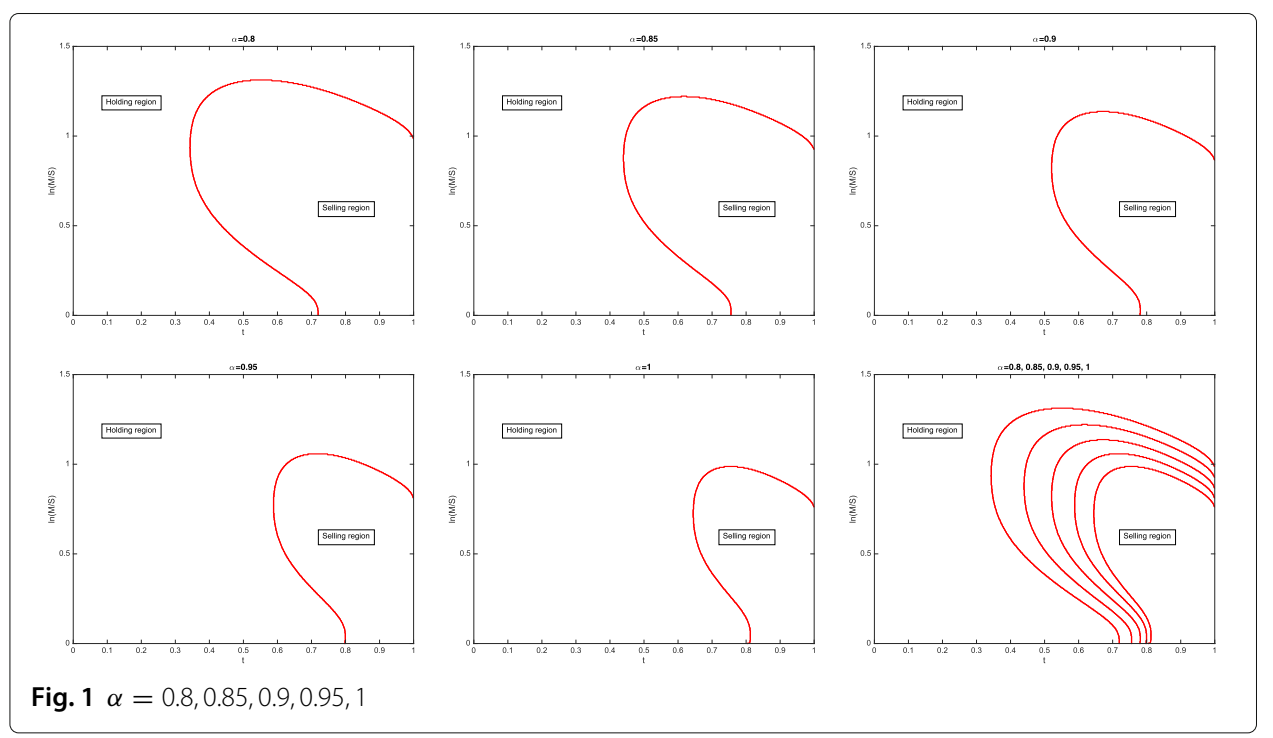




\section{Conclusion}

This paper considers an optimal stopping time point for the investor who is expected to maximize her personal utilities and to minimize the difference between the realized return at the stopping point and her potentially maximum return. Our utility function of a quadratic form is more general than that of a fraction form where the denominator may be zero in Shiryaev et al. (2008). Furthermore, our investment problem, which includes the portfolio in the drift and volatility terms of the dynamic systems, makes the problem including multi-dimensional financial assets more realistic and meaningful.

\section{Appendix A: expression of function $\boldsymbol{G}_{\mathbf{1}}$}

We now derive the explicit expression of the function $G_{1}$, defined by

$$
\begin{aligned}
G_{1}(t, y) & =\mathbb{E}\left[\exp \left(\max \left\{y, \max _{0 \leq u \leq T-t}(v u+B(u))\right\}\right)\right] \\
& =\int_{y}^{\infty} e^{z} \mathrm{~d} \mathbb{P}\left(\max _{0 \leq u \leq T-t}(\nu u+B(u)) \leq z\right)+e^{y} \mathbb{P}\left(\max _{0 \leq u \leq T-t}(\nu u+B(u)) \leq y\right) .
\end{aligned}
$$

Note that

$$
\mathbb{P}\left(\max _{0 \leq u \leq T-t}(v u+B(u)) \leq z\right)=\Phi\left(\frac{z-v(T-t)}{\sqrt{T-t}}\right)-e^{2 v z} \Phi\left(\frac{-z-v(T-t)}{\sqrt{T-t}}\right) .
$$

According to the standard normal distribution, we have

$$
\begin{aligned}
\int_{y}^{\infty} e^{z} \mathrm{~d} \Phi\left(\frac{z-v(T-t)}{\sqrt{T-t}}\right) & =\int_{y}^{\infty} e^{z} \frac{1}{\sqrt{2 \pi(T-t)}} e^{-\frac{(z-v(T-t))^{2}}{2(T-t)}} \mathrm{d} z \\
& =e^{\left(v+\frac{1}{2}\right)(T-t)}\left[1-\Phi\left(\frac{y-(v+1)(T-t)}{\sqrt{T-t}}\right)\right] .
\end{aligned}
$$

Assume that $v \neq-\frac{1}{2}$. Then

$$
\begin{aligned}
& \int_{y}^{\infty} e^{z} \mathrm{~d}\left[e^{2 v z} \Phi\left(\frac{-z-v(T-t)}{\sqrt{T-t}}\right)\right] \\
= & \int_{y}^{\infty} 2 v e^{(1+2 v) z} \Phi\left(\frac{-z-v(T-t)}{\sqrt{T-t}}\right) \mathrm{d} z+\int_{y}^{\infty} e^{(1+2 v) z} \mathrm{~d} \Phi\left(\frac{-z-v(T-t)}{\sqrt{T-t}}\right) \\
= & -\frac{2 v}{1+2 v} e^{(1+2 v) y} \Phi\left(\frac{-y-v(T-t)}{\sqrt{T-t}}\right)-\frac{1}{1+2 v} e^{\left(v+\frac{1}{2}\right)(T-t)}\left[1-\Phi\left(\frac{y-(v+1)(T-t)}{\sqrt{T-t}}\right)\right] .
\end{aligned}
$$

Thus

$$
\begin{aligned}
G_{1}(t, y)= & e^{y} \Phi\left(\frac{y-v(T-t)}{\sqrt{T-t}}\right)-\frac{1}{1+2 v} e^{(1+2 v) y} \Phi\left(\frac{-y-v(T-t)}{\sqrt{T-t}}\right) \\
& +\frac{2(1+v)}{1+2 v} e^{\left(v+\frac{1}{2}\right)(T-t)}\left[1-\Phi\left(\frac{y-(v+1)(T-t)}{\sqrt{T-t}}\right)\right] .
\end{aligned}
$$

In addition, note that when $v=-\frac{1}{2}$,

$$
\begin{aligned}
& \int_{y}^{\infty} e^{z} \mathrm{~d}\left[e^{2 v z} \Phi\left(\frac{-z-v(T-t)}{\sqrt{T-t}}\right)\right] \\
= & \int_{y}^{\infty} e^{z} \mathrm{~d}\left[e^{-z} \Phi\left(\frac{-z-v(T-t)}{\sqrt{T-t}}\right)\right] \\
= & -\int_{y}^{\infty} \Phi\left(\frac{-z-v(T-t)}{\sqrt{T-t}}\right) \mathrm{d} z+\int_{y}^{\infty} \mathrm{d} \Phi\left(\frac{-z-v(T-t)}{\sqrt{T-t}}\right) \\
= & y \Phi\left(\frac{-y-v(T-t)}{\sqrt{T-t}}\right)-\frac{\sqrt{T-t}}{\sqrt{2 \pi}} e^{-\frac{(x+v(T-t))^{2}}{2(T-t)}}+v(T-t)\left[1-\Phi\left(\frac{y+v(T-t)}{\sqrt{T-t}}\right)\right] \\
& -\Phi\left(\frac{-y-v(T-t)}{\sqrt{T-t}}\right) .
\end{aligned}
$$


Thus

$$
\begin{aligned}
G_{1}(t, y)= & 1-\Phi\left(\frac{y-(v+1)(T-t)}{\sqrt{T-t}}\right)-y \Phi\left(\frac{-y-v(T-t)}{\sqrt{T-t}}\right)+\frac{\sqrt{T-t}}{\sqrt{2 \pi}} e^{-\frac{(y+v(T-t))^{2}}{2(T-t)}} \\
& -v(T-t)\left[1-\Phi\left(\frac{y+v(T-t)}{\sqrt{T-t}}\right)\right]+e^{y} \Phi\left(\frac{y-v(T-t)}{\sqrt{T-t}}\right) .
\end{aligned}
$$

\section{Appendix B: expression of function $\mathbf{G}_{\mathbf{2}}$}

We now derive the explicit expression of the function $G_{2}$, defined by

$$
\begin{aligned}
G_{2}(t, y)= & \mathbb{E}\left[\exp \left(\max \left\{y, \max _{0 \leq u \leq T-t}(v u+B(u))\right\}\right)^{2}\right] \\
= & \int_{y}^{\infty} e^{2 z} \mathrm{~d} \mathbb{P}\left(\max _{0 \leq u \leq T-t}(v u+B(u)) \leq z\right) \\
& +e^{2 y} \mathbb{P}\left(\max _{0 \leq u \leq T-t}(v u+B(u)) \leq y\right) .
\end{aligned}
$$

Note that

$$
\mathbb{P}\left(\max _{0 \leq u \leq T-t}(v u+B(u)) \leq z\right)=\Phi\left(\frac{z-v(T-t)}{\sqrt{T-t}}\right)-e^{2 v z} \Phi\left(\frac{-z-v(T-t)}{\sqrt{T-t}}\right) .
$$

According to the standard normal distribution, we have

$$
\begin{aligned}
\int_{y}^{\infty} e^{2 z} \mathrm{~d} \Phi\left(\frac{z-v(T-t)}{\sqrt{T-t}}\right) & =\int_{y}^{\infty} e^{2 z} \frac{1}{\sqrt{2 \pi(T-t)}} e^{-\frac{(z-v(T-t))^{2}}{2(T-t)}} \mathrm{d} z \\
& =e^{2(v+1)(T-t)}\left[1-\Phi\left(\frac{y-(v+2)(T-t)}{\sqrt{T-t}}\right)\right] .
\end{aligned}
$$

Assume that $v \neq-1$. Then

$$
\begin{aligned}
& \int_{y}^{\infty} e^{2 z} \mathrm{~d}\left[e^{2 v z} \Phi\left(\frac{-z-v(T-t)}{\sqrt{T-t}}\right)\right] \\
= & \int_{y}^{\infty} 2 v e^{2(1+v) z} \Phi\left(\frac{-z-v(T-t)}{\sqrt{T-t}}\right) \mathrm{d} z+\int_{x}^{\infty} e^{2(1+v) z} \mathrm{~d} \Phi\left(\frac{-z-v(T-t)}{\sqrt{T-t}}\right) \\
= & -\frac{v}{1+\nu} e^{2(1+v) y} \Phi\left(\frac{-y-v(T-t)}{\sqrt{T-t}}\right)-\frac{1}{1+v} e^{2(v+1)(T-t)}\left[1-\Phi\left(\frac{y-(v+2)(T-t)}{\sqrt{T-t}}\right)\right] .
\end{aligned}
$$

Thus

$$
\begin{aligned}
G_{2}(t, y)= & e^{2 y} \Phi\left(\frac{y-v(T-t)}{\sqrt{T-t}}\right)-\frac{1}{1+2 v} e^{2(1+v) y} \Phi\left(\frac{-y-v(T-t)}{\sqrt{T-t}}\right) \\
& +\frac{2+v}{1+\nu} e^{2(v+1)(T-t)}\left[1-\Phi\left(\frac{y-(v+2)(T-t)}{\sqrt{T-t}}\right)\right] .
\end{aligned}
$$

Also, note that when $v=-1$,

$$
\begin{aligned}
& \int_{y}^{\infty} e^{2 z} \mathrm{~d}\left[e^{2 v z} \Phi\left(\frac{-z-v(T-t)}{\sqrt{T-t}}\right)\right] \\
= & \int_{y}^{\infty} e^{2 z} \mathrm{~d}\left[e^{-2 z} \Phi\left(\frac{-z-v(T-t)}{\sqrt{T-t}}\right)\right] \\
= & -2 \int_{y}^{\infty} \Phi\left(\frac{-z-v(T-t)}{\sqrt{T-t}}\right) \mathrm{d} z+\int_{y}^{\infty} \mathrm{d} \Phi\left(\frac{-z-v(T-t)}{\sqrt{T-t}}\right) \\
= & 2 y \Phi\left(\frac{-y-v(T-t)}{\sqrt{T-t}}\right)-\frac{2 \sqrt{T-t}}{\sqrt{2 \pi}} e^{-\frac{(y+v(T-t))^{2}}{2(T-t)}}+2 v(T-t)\left[1-\Phi\left(\frac{y+v(T-t)}{\sqrt{T-t}}\right)\right] \\
& -\Phi\left(\frac{-y-v(T-t)}{\sqrt{T-t}}\right) .
\end{aligned}
$$


Thus

$$
\begin{aligned}
G_{2}(t, y)= & 1-\Phi\left(\frac{y-(v+2)(T-t)}{\sqrt{T-t}}\right)-2 y \Phi\left(\frac{-y-v(T-t)}{\sqrt{T-t}}\right)+\frac{2 \sqrt{T-t}}{\sqrt{2 \pi}} e^{-\frac{(y+v(T-t))^{2}}{2(T-t)}} \\
& -2 v(T-t)\left[1-\Phi\left(\frac{y+v(T-t)}{\sqrt{T-t}}\right)\right]+e^{2 y} \Phi\left(\frac{y-v(T-t)}{\sqrt{T-t}}\right)
\end{aligned}
$$

Funding

This work is supported by Research Grants Council of Hong Kong under grant no. 519913 and 15224215, and National Natural Science Foundation of China (No.11571124).

Authors' contributions

All authors read and approved the final manuscript.

Competing interests

The authors declare that they have no competing interests.

\section{Publisher's Note}

Springer Nature remains neutral with regard to jurisdictional claims in published maps and institutional affiliations.

\section{Author details}

${ }^{1}$ Department of Applied Mathematics, The Hong Kong Polytechnic University, Hong Kong, China. ${ }^{2}$ School of Mathematical Sciences, South China Normal University, Guangzhou, China.

Received: 8 October 2017 Accepted: 2 November 2017

Published online: 17 November 2017

\section{References}

Ceci C, Bassan B (2004) Mixed optimal stopping and stochastic control problems with semicontinuous final reward for diffusion processes. Stochast Stochast Rep 76:323-337

Chang MH, Pang T, Yong J (2009) Optimal stopping problem for stochastic differential equations with random coefficients. SIAM J Control Optim 48:941-971

Choi K, Koo H, Kwak D (2004) Optimal stopping of active portfolio management. Ann Econ Finance 5:93-126

Dayanik S, Karatzas I (2003) On the optimal stopping problem for one-dimensional diffusions. Stochast Process Appl 107:173-212

Li D, Ng WL (2000) Optimal dynamic portfolio selection: Multi-period means-variance formulation. Math Financ 10:387-406

Li X, Zhou XY (2006) Continuous-time mean-variance efficiency: The 80\% rule. Ann Appl Probab 16:1751-1763 Markowitz H (1952) Portfolio selection. J Finance 7:77-91

Markowitz, H (1959) Portfolio Selection: Efticient Diversification of Investments

Merton RC (1969) Lifetime portfolio selection under uncertainty: The continuous-time case. Rev Econ Stat 51:247-257

Merton, RC (1971) Optimum consumption and portfolio rules in a continuous-time model. J Econ Theory 3:373-413

Merton, RC (1973) Theory of rational option pricing. Bell J Econ Manag Sci 4:141-183

Shiryaev A, Xu ZQ, Zhou XY (2008) Thou shalt buy and hold. Quant Finan 8:765-776

Zhou XY, Li D (2000) Continuous-time mean-variance portfolio selection: A stochastic LQ framework. Appl Math Optim 42:19-33

\section{Submit your manuscript to a SpringerOpen ${ }^{\circ}$ journal and benefit from:}

- Convenient online submission

Rigorous peer review

- Open access: articles freely available online

- High visibility within the field

- Retaining the copyright to your article

Submit your next manuscript at $\gg$ springeropen.com 